\title{
Umfassende Selbstbesinnung und neues Image: ein intensives 2015
}

\section{Anne-Geneviève Bütikofer}

Lic. iur., Generalsekretärin der FMH

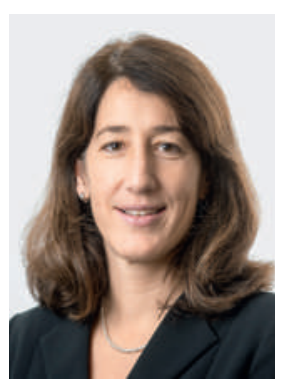

Jedes Jahr birgt für das Generalsekretariat (GS) wie für die gesamte FMH eine Reihe von Herausforderungen und bringt neue Vorhaben mit sich, die uns einiges an Energie, Engagement und Hartnäckigkeit abverlangen. Erst zum Jahresende erschliesst sich uns das tatsächliche Ausmass der geleisteten Arbeit und der ausgefochtenen Kämpfe.

Das Jahr 2015 war für das GS und seine Mitarbeitenden vor allem durch die von der Ärztekammer im Herbst 2014 beschlossene Budgetstabilisierung geprägt. Diese Aufgabe bot Gelegenheit, die eigene Tätigkeit auf allen Ebenen der FMH und ihrer Strukturen umfassend zu überdenken und den Wandel unserer Organisation stärker voranzutreiben.

Dieses Jahr haben wir die symbolische Schallmauer von 40000 Mitgliedern durchbrochen.

Aber was haben wir - abgesehen von der Überprüfung, ob unsere Tätigkeit mit unseren Finanzen in Einklang steht, und der Festlegung unserer Prioritäten - 2015 eigentlich gemacht? Wir haben die Verwandlung fortgesetzt, um unser Dienstleistungsangebot zu verbessern und unsere Funktionsweise zu optimieren. Über die Umsetzung der im Vorjahr entwickelten Massnahmen zur Finanzkontrolle hinaus haben wir unsere Finanzstrategie analysiert, unsere Liquiditätsplanung überdacht sowie die Zusammenarbeit und finanzielle Unterstützung von Partnerinstitutionen überprüft. Weiterhin haben wir unseren Führungskräften leistungsfähigere Tools zur Verfügung gestellt, die ihnen eine bessere Arbeitsplanung und genauere Zielverfolgung erleichtern, und einen ersten Fortbildungszyklus im Managementbereich für sie organisiert. Darüber hinaus ist derzeit das neue Risikomanagement, das wir nicht nur auf finanzieller, sondern auch auf operativer und strategischer Ebene anwenden wollen, ebenfalls auf dem Weg. Im Vorfeld einer solchen Analyse muss natürlich sichergestellt sein, dass entsprechende Prozesse tatsächlich vorhanden und angemessen sind. Dies wird in Zukunft gewährleisten, dass unsere Organisation unabhängig von den Unwägbarkeiten des Alltags jederzeit funktionsfähig ist. Dafür ist es auch notwendig, dass auf Ebene des Generalsekretariats der Datenschutz sicher- gestellt und ein Konzept zur Einhaltung der gesetzlichen Vorgaben vorhanden ist.

Neben diesen strukturellen Arbeiten im Hintergrund verloren wir nie unser Ziel aus den Augen: Wir wollen das Image unseres Verbands und den Bekanntheitsgrad der Leistungen für unsere nunmehr 40000 Mitglieder stärken - diese symbolische Schallmauer wurde dieses Jahr durchbrochen. In diesem Zusammenhang präsentieren wir uns ab sofort mit einem moderneren und verjüngten Logo. Schritt für Schritt werden wir im Zeichen dieses neuen Auftritts die Leistungen für unsere Mitglieder ausbauen. So haben wir in diesem Jahr erstmals einen umfassenden Dienstleistungskatalog veröffentlicht, den Zugriff auf die Plattform myFMH vereinfacht und unseren Organen und deren Delegierten ein modernes, interaktives Arbeits- und Kommunikationstool bereitgestellt, das noch weiterentwickelt werden soll.

Kurz: Über einen Mangel an Arbeit konnten wir uns nicht beklagen. In den letzten Jahrzehnten hat sich die Berufswelt in schwindelerregender Weise weiterentwickelt. Heute spricht man schon nicht mehr von "Change Management", sondern von "New Change Management»: Ständige Veränderung ist zur Normalität geworden. Die Arbeitswelt wird immer komplexer, der Wettbewerb immer härter, die finanziellen Vorgaben immer strikter, die gesetzlichen und aufsichtsrechtlichen Anforderungen immer zahlreicher, die Erwartungen der Interessengruppen (Kunden, Patienten, Mitglieder) immer höher und sprunghafter, die einzu-

Erst zum Jahresende erschliesst sich uns das tatsächliche Ausmass der geleisteten Arbeit und der ausgefochtenen Kämpfe.

richtenden Kontrollen immer genauer und die Technologien entwickeln sich immer schneller. Damit wir diese Komplexität bewältigen und dabei glaubwürdig bleiben können, muss sich unser Geschäftsführungsmodell anpassen. Für diese Ziele wird sich das GS weiter einsetzen, ebenso wie für die so wichtigen Themendossiers, mit denen wir die Interessen der Ärzteschaft verteidigen.

Abschliessend möchte ich den Mitarbeitenden im GS der FMH sowie den Leserinnen und Lesern der SÄZ schöne und erholsame Festtage wünschen. 